\title{
Bats, objectivity, and viral spillover risk
}

\author{
Beckett Sterner $^{1}$ (D) Steve Elliott ${ }^{2} \cdot$ Nate Upham $^{1} \cdot$ Nico Franz $^{1}$
}

Received: 2 September 2020 / Accepted: 7 January 2021 / Published online: 13 January 2021 (C) Springer Nature Switzerland AG 2021

\begin{abstract}
What should the best practices be for modeling zoonotic disease risks, e.g. to anticipate the next pandemic, when background assumptions are unsettled or evolving rapidly? This challenge runs deeper than one might expect, all the way into how we model the robustness of contemporary phylogenetic inference and taxonomic classifications. Different and legitimate taxonomic assumptions can destabilize the putative objectivity of zoonotic risk assessments, thus potentially supporting inconsistent and overconfident policy decisions.
\end{abstract}

Keywords Special reservoir hypothesis $\cdot$ Zoonotic disease $\cdot$ Covid-19 $\cdot$ Viral spillover

With the outbreak of the global Covid-19 pandemic, policy makers are seeking to prevent similar outbreaks of viral zoonoses in the future (Carlson 2020). ${ }^{1}$ Proposed policy interventions, however, are frequently imbued with fears about particular vectors, such as bats, and how they interact with human cultural practices (Zhao 2020). Scientists have also developed competing and rapidly changing models for what caused the pandemic and how it will unfold, complicating the pursuit of evidencebased policy. What guidelines should scientists follow in modeling zoonotic disease risks to inform decision-making when background assumptions are unsettled or rapidly evolving? This challenge runs deeper than one might expect, all the way into

\footnotetext{
${ }^{1}$ This note belongs to the Topical Collection "Seeing Clearly Through COVID-19: Current and future questions for the history and philosophy of the life sciences", edited by G. Boniolo and L. Onaga.
}

Beckett Sterner

beckett.sterner@asu.edu

1 School of Life Sciences, Arizona State University, Tempe, USA

2 Center for Gender Equity in Science and Technology, Arizona State University, Tempe, USA 
basic theories related to robust modeling of phylogenetic inferences and taxonomic classifications.

Here, we explore the implications of taxonomic and phylogenetic knowledge representation for the novel Special Reservoir Hypothesis (SRH) to show how different assumptions can generate different risk assessments (Brook and Dobson 2015; Mollentze and Streicker 2020). Amid public perceptions that some taxa naturally pose high risks, the SRH raises the bar for supporting evidence by formulating a precise statistical expectation for how many viral spillovers we'd expect to find from a taxonomic group by chance. The hypothesis posits ecological or physiological causes for variations in the corresponding risk of zoonotic transmission from those taxa. It's also subject to controversy: Mollentze and Streicker (2020) conclude that there is no support for the SRH, so that groups such as bats (Chiroptera) do not in fact pose a higher risk than expected by chance, but their result contradicts consensus support from prior studies for bats and rodents as special reservoirs, e.g. (Guy et al. 2019). Researchers participating in this debate position it as key to informing broader risk management strategy, including whether to target surveillance on hosts or sites (e.g. wild bats or rural villages).

Differing taxonomic and phylogenetic assumptions, each legitimate, can destabilize the putative objectivity of zoonotic risk assessments, thus potentially supporting incompatible policy decisions. Testing the SRH requires comparisons across the focal, recognized taxa as well as careful modeling of their evolutionary relationships considering differences in data-source quality. Phylogenetic inference helps researchers quantify the evolutionary distances from humans to other animal taxa as potential hurdles for viruses leaping to humans. A taxonomic classification provides researchers a background theory by which to integrate data, for example by defining the number of bat species and providing species names that researchers use to query databases of prior viral research. Systematic biologists continually update and dispute mammal phylogeny and taxonomy, including the latest species-level phylogeny (Upham et al. 2019) and an increase in recognized living species from 5416 to 6399 in the last 15 years (Burgin et al. 2018). Therefore, zoonotic risk modellers aiming to test the SRH face a recurring need to account for the influence that their choice of phylogenetic and taxonomic theories has on modeling results. We illustrate this issue using two input variables for contemporary models testing the SRH: how many species each taxonomic group of interest contains, and how much research effort scientists have devoted to studying viruses in each of those groups.

One's choice of background theories (phylogeny and taxonomy) impacts inferences about the extent of viral zoonoses hosted within different species groups. In a reanalysis of prior results using improved phylogenies and comparative methods, Guy et al. (2019) showed that the choice of phylogeny influences which variables appear in the best model for predicting zoonoses in bats (though not in rodents). They found that when modelling risks with one phylogeny, the best model included 
a variable for species range size but not one for life-history traits (such as hibernation or torpor), while modelling with a different phylogeny returned the opposite result (similar $\mathrm{R}^{2}$ values of 0.29 and 0.32 , respectively). Thus, the choice of phylogeny yielded alternative causal explanations in support of the SRH, and ultimately different suggestions for policy interventions.

Furthermore, taxonomists' preferences for one species concept over others might influence species counts. For instance, the number of recognized mammal species grew by 55\% between 1982 and 2018 (Burgin et al. 2018), which reflects the widespread adoption of DNA-based evidence over morphology alone and increased use of the phylogenetic species concept over the coarser-grained biological species concept (Zachos 2018). For the SRH, species counts set a group's expected baseline for zoonotic diseases, so uneven (and often insufficiently specified) preferences of species concepts across groups may bias results. To our knowledge this issue remains unexplored.

Choice of phylogeny and taxonomy can also impact estimates of how much research attention different viral reservoir groups have received. Leading models have consistently found that the number of publications or citations for viruses and their host reservoirs groups is among the most important predictors of zoonotic disease risk, e.g. (Brook and Dobson 2015; Guy et al. 2019; Mollentze and Streicker 2020). These variables are based on querying databases such as PubMed using taxonomic names and synonym lists. Synonymy, however, varies across classifications: bats have 1436 synonyms listed at itis.gov versus 760 at iucnredlist.org, as of September 1,2020 . Including synonyms in database queries can have a large impact on results, e.g. increasing search results by $16 \%$ for animal species in PubMed (Guala 2016), so changes in the background taxonomy should similarly influence how articles are aggregated to potential virus or reservoir groups. Single taxonomic names also frequently change in meaning across classifications (i.e. the set of included organisms), sometimes dramatically (Franz et al. 2016), which can't be measured or accommodated using synonyms alone.

To achieve objective estimates of viral spillover risks, then, scientists cannot assume that taxonomy and phylogeny are stable, universal, or neutral assumptions across studies. What might they do instead? One option is to redouble efforts to eliminate dependence on contested or uncertain assumptions, for example by providing tools for other researchers to reproduce or test the robustness of the modeling process. A second option is to direct attention to lower-ranking taxa that are distributed within the jurisdictions of particular decision makers or are relevant to local contexts, for instance particular ecosystems or markets for wildlife, food, and animal products. Either way, more objective risk estimates may still lead to unfair treatment for cultures that interact with any taxa found to pose special risks (Fig. 1). 
Fig. 1 Predicted versus observed number of species in taxonomic orders of animals that have been sources of zoonotic viral diseases (Mollentze and Streicker 2020,

Fig. 4E). Model results show no evidence that some groups pose a significantly elevated risk of future zoonoses, contradicting results from several prior studies. Figure is Copyright (2020) National Academy of Sciences.

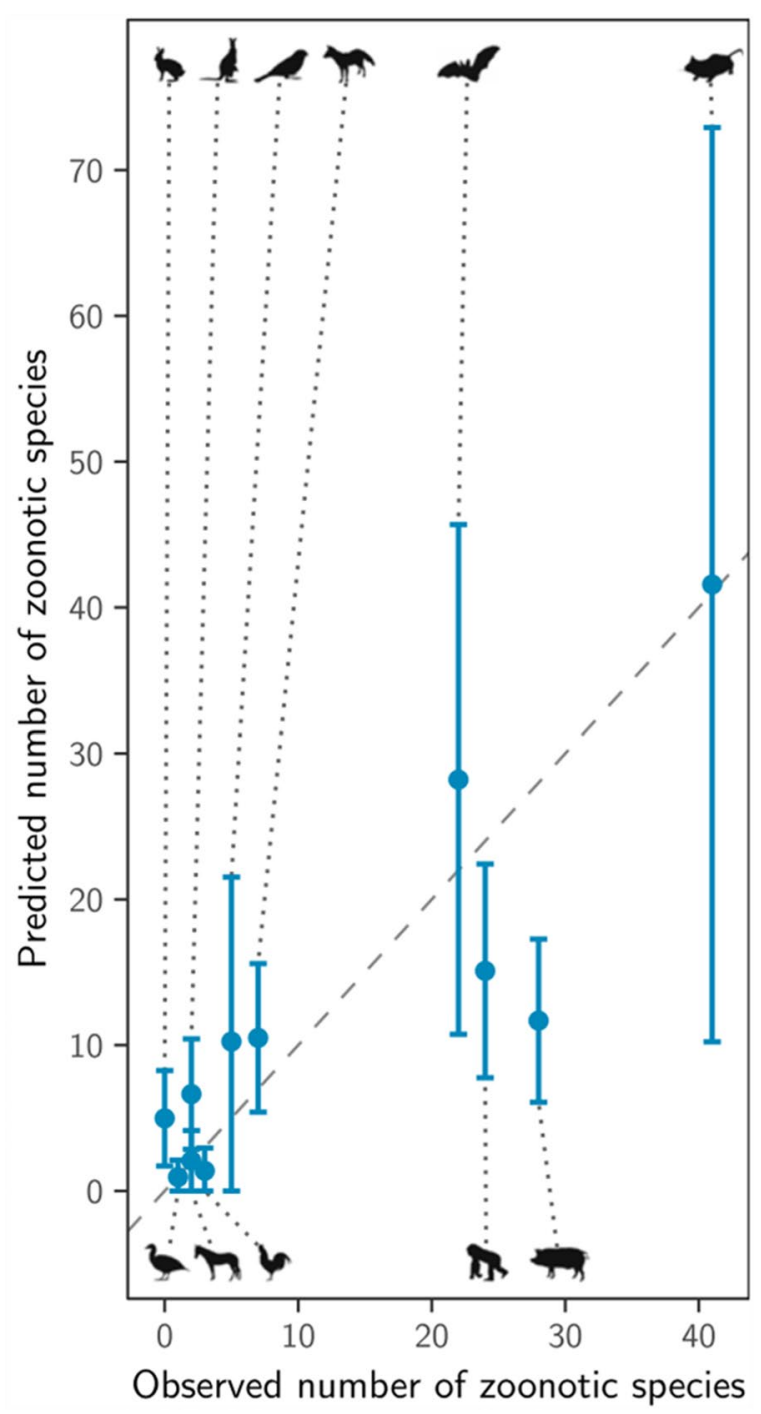

\section{References}

Brook, C. E., \& Dobson, A. P. (2015). Bats as 'Special' reservoirs for emerging zoonotic pathogens. Trends in Microbiology, 23(3), 172-180. https://doi.org/10.1016/j.tim.2014.12.004.

Burgin, C. J., Colella, J. P., Kahn, P. L., \& Upham, N. S. (2018). How many species of mammals are there? Journal of Mammalogy, 99(1), 1-14. https://doi.org/10.1093/jmammal/gyx 147.

Carlson, C. J. (2020). From PREDICT to prevention, one pandemic later. The Lancet Microbe. https:// doi.org/10.1016/S2666-5247(20)30002-1.

Franz, N. M., Pier, N. M., Reeder, D. M., Chen, M., Shizhuo, Yu., Kianmajd, P., et al. (2016). Two influential primate classifications logically aligned. Systematic Biology, 65(4), 561-582. https:// doi.org/10.1093/sysbio/syw023. 
Guala, G. F. (2016). The importance of species name synonyms in literature searches. PLoS ONE, 11(9), e0162648. https://doi.org/10.1371/journal.pone.0162648.

Guy, C., Thiagavel, J., Mideo, N., \& Ratcliffe, J. M. (2019). Phylogeny matters: Revisiting 'a comparison of bats and rodents as reservoirs of zoonotic viruses.' Royal Society Open Science, 6(2), 181182. https://doi.org/10.1098/rsos.181182.

Mollentze, N., \& Streicker, D. G. (2020). Viral Zoonotic Risk Is Homogenous among Taxonomic Orders of Mammalian and Avian Reservoir Hosts. Proceedings of the National Academy of Sciences. https://doi.org/10.1073/pnas.1919176117.

Upham, N. S., Esselstyn, J. A., \& Jetz, W. (2019). Inferring the mammal tree: species-level sets of phylogenies for questions in ecology, evolution, and conservation. PLOS Biology, 17(12), e3000494. https://doi.org/10.1371/journal.pbio.3000494.

Zachos, F. E. (2018). Mammals and meaningful taxonomic units: the debate about species concepts and conservation. Mammal Review, 48(3), 153-159. https://doi.org/10.1111/mam.12121.

Zhao, H. (2020). COVID-19 drives new threat to bats in China. Science, 367(6485), 1436-1436. https:// doi.org/10.1126/science.abb3088.

Publisher's Note Springer Nature remains neutral with regard to jurisdictional claims in published maps and institutional affiliations. 